University of Nebraska - Lincoln

DigitalCommons@University of Nebraska - Lincoln

$5-1-2001$

\title{
Nucleation of Water and Methanol Droplets on Cations and Anions: The Sign Preference
}

\author{
K.J. Oh \\ University of Nebraska-Lincoln \\ G.T. Gao \\ University of Nebraska-Lincoln \\ Xiao Cheng Zeng \\ University of Nebraska-Lincoln, xzeng1@unl.edu
}

Follow this and additional works at: https://digitalcommons.unl.edu/chemzeng

Part of the Chemistry Commons

Oh, K.J.; Gao, G.T.; and Zeng, Xiao Cheng, "Nucleation of Water and Methanol Droplets on Cations and Anions: The Sign Preference" (2001). Xiao Cheng Zeng Publications. 46.

https://digitalcommons.unl.edu/chemzeng/46

This Article is brought to you for free and open access by the Published Research - Department of Chemistry at DigitalCommons@University of Nebraska - Lincoln. It has been accepted for inclusion in Xiao Cheng Zeng Publications by an authorized administrator of DigitalCommons@University of Nebraska - Lincoln. 


\title{
Nucleation of Water and Methanol Droplets on Cations and Anions: The Sign Preference
}

\author{
K. J. Oh, G. T. Gao, and X. C. Zeng \\ Department of Chemistry and Center for Materials Research and Analysis, University of Nebraska-Lincoln, \\ Lincoln, Nebraska 68588 \\ (Received 7 June 2000)
}

\begin{abstract}
The barrier height to cation- and anion-induced nucleation to produce water and methanol droplets are calculated by means of an umbrella-sampling Monte Carlo method. The computer simulation corroborates the century-old finding of Wilson that the anion is a better nucleator to produce water droplets than the cation having the same magnitude of charge, even without the presence of external electric field. The simulation also shows that the cation is a better nucleator to produce methanol droplets than the anion.
\end{abstract}

DOI: 10.1103/PhysRevLett.86.5080

Nucleation of water vapor on ions plays an important role in many atmospheric processes [1-4], e.g., generation of aerosols in the upper atmosphere and the formation of acid rain. Knowledge about prenucleation embryos also has implications concerning the structure of ionic liquids [5]. It is now well known that ions promote water vapor condensation, irrespective of the sign of ions. However, the asymmetry between water-positive-ion and waternegative-ion interactions can lead to ion-sign preference in condensation of water. This phenomenon is known as the sign preference [6-13].

In 1897, Wilson observed the dependence of the watercondensation rate on the sign of ions in the expansioncloud-chamber experiments [6]. He found negative ions were more effective to produce a rain of water droplets in the presence of an external electric field. Ninety years later Rabeony and Mirabel reported that the sign preference of water was not detectable [9] if the external electric field was not applied. To date, the existence (or disappearance) of the sign preference in the condensation of water without the presence of an external electric field is still not fully confirmed (see Ref. [13]).

Theoretically, prediction of the sign preference requires knowledge of barrier height to cation- and anion-induced condensation [14]. The classical theory of nucleation $[15,16]$ is unable to differentiate between the effects of cation and anion on water condensation. Although the thermodynamic model developed by Rusanov and Kuni [17] can differentiate the sign preference, the obtained sign effect is very sensitive to an input parameter. The modern density-functional theory (DFT) of nucleation $[18,19]$ does predict a sign preference for simple dipolar molecules. However, incorporation of molecular structures of hydrogen-bond molecules in DFT requires many approximations [20] which render calculation of barrier to nucleation semiquantitative. To accurately predict the sign preference of hydrogen-bonding systems, a combined molecular-theory/computer-simulation approach [21] is called for. In this Letter, we report computer simulation results of barrier to the cation- and anion-induced water and methanol condensation in the absence of an external electric field. We note that for methanol experimentally
PACS numbers: 64.60.Qb, 82.60.Nh, 92.60.Jq, 92.60.Ls

measured rates of ion-induced nucleation have been reported [13].

We used a recently developed small-ensemble umbrellasampling Monte Carlo (MC) method [22] to compute the free energy of cluster formation and to determine barrier height to ion-induced nucleation. This MC method is based on the physical cluster theory [21]; namely, a supersaturated vapor is viewed as a mixture of physical clusters, and a configuration of supersaturated vapor is described by the cluster-size distribution $\left\{n_{i}^{w}, n_{i}^{s}\right\}$, where $n_{i}^{w}$ and $n_{i}^{s}$ are the number of clusters containing $i$ molecules, and 0 and 1 ion, respectively. The total number of ion-containing clusters is $N_{s}=\sum_{i=0} n_{i}^{s}$. Since typically the density of ions involved in the cloud-chamber experiments is extremely low, it is reasonable to assume that a cluster contains one ion at most. Intercluster interactions can also be neglected [21,23]. Maximizing the partition function of the supersaturated vapor gives the most probable cluster size distribution $n_{i}^{w}=q_{i}^{w} e^{\mu_{i}^{w}\left(p_{i}^{w}\right) / k_{B} T}$ and $n_{i}^{s}=q_{i}^{s} e^{\mu_{i}^{s}\left(p_{i}^{s}\right) / k_{B} T}$, where $k_{B}$ is the Boltzmann constant, $T$ is the temperature, $\mu_{i}^{w}\left(p_{i}^{w}\right)$ and $\mu_{i}^{s}\left(p_{i}^{s}\right)$ are the chemical potential of pure cluster and ion-containing cluster at their partial pressure $p_{i}^{w}$ and $p_{i}^{s}$, respectively, and $q_{i}^{w}$ and $q_{i}^{s}$ are the corresponding partition functions. Other by-products of the maximization are the laws of mass action $\mu_{i}^{w}\left(p_{i}^{w}\right)=i \mu_{1}^{w}\left(p_{1}^{w}\right), \quad \mu_{i}^{s}\left(p_{i}^{s}\right)=i \mu_{1}^{w}\left(p_{1}^{w}\right)+\mu_{0}^{s}\left(p_{0}^{s}\right)$, and $\mu_{1}^{w}\left(p_{1}^{w}\right)=\mu_{1}^{s}\left(p_{1}^{s}\right)-\mu_{0}^{s}\left(p_{0}^{s}\right)$.

The free energy of cluster formation is defined as $\Delta G_{i}^{s}=\mu_{i}^{s}\left(p_{1}^{w}\right)-\mu_{0}^{s}\left(p_{1}^{w}\right)-i \mu_{1}^{w}\left(p_{1}^{w}\right)$. Using the ideal gas relations $\mu_{i}^{s}\left(p_{1}^{w}\right)=\mu_{i}^{s}\left(p_{i}^{s}\right)+k_{B} T \ln n_{1}^{w} / n_{i}^{s}$ and $\mu_{0}^{s}\left(p_{1}^{w}\right)=\mu_{0}^{s}\left(p_{0}^{s}\right)+k_{B} T \ln n_{1}^{w} / n_{0}^{s}$ as well as the laws of mass action, $\Delta G_{i}^{s}$ can be given as

$$
\Delta G_{i}^{s}=k_{B} T \ln \frac{n_{0}^{s}}{n_{i}^{s}}=k_{B} T \ln \frac{n_{0}^{s} / N_{s}}{n_{i}^{s} / N_{s}},
$$

where

$$
N_{s}=\sum_{i=0}^{i_{\max }} n_{i}^{s}=\sum_{i=0}^{i_{\max }} q_{i}^{s} e^{i \mu_{1}^{w}\left(p_{1}^{w}\right) / k_{B} T} e^{\mu_{0}^{s}\left(p_{0}^{s}\right) / k_{B} T} .
$$

In Eq. (2), $i_{\max }$ is an upper bound of cluster size which is used to constrain the supersaturated vapor into a metastable equilibrium state; $N_{s}$ can be viewed as a grand canonical 
TABLE I. Density (in units of $10^{-6} \AA^{-3}$ ) of supersaturated and saturated (at $298.15 \mathrm{~K}$ ) vapor, supersaturation, magnitude of charge and size of model ions, and difference of free-energy barrier for different water models.

\begin{tabular}{lclclcr}
\hline \hline Water model & $\rho$ & $\rho_{0}{ }^{29}$ & $S \approx \rho / \rho_{0}$ & $q$ & $\sigma(\AA)$ & $\delta\left(k_{B} T\right)$ \\
\hline TIP4P & 4.0 & 1.4 & 2.9 & $0.6 e$ & 4.75 & -4.96 \\
SPC/E & 0.75 & 0.25 & 3.0 & $1 e$ & 8.0 & -1.06 \\
Dang-Chang & 10.4 & $\cdots$ & $\cdots$ & $1 e$ & 4.75 & -5.82 \\
\hline \hline
\end{tabular}

partition function. The probability of finding an ioncontaining cluster of size $i, n_{i}^{s} / N_{s}$, can be evaluated by combining the grand canonical ensemble MC method with umbrella-sampling technique $[22,23]$.

We employed the TIP4P model water [24] and OPLS model methanol [25]. The model cation or anion is a Lennard-Jones (LJ) sphere (with LJ parameters [26] $\epsilon=$ $0.0014 \mathrm{~kJ} / \mathrm{mol}$ and $\sigma=4.75 \AA$ ) containing a point charge $q=0.6 e$. To assure that our final conclusion is model independent, we also examined two other water models, SPC/E [27] and Dang-Chang polarizable model [28], and a model ion with a greater charge (see Table I). In the MC simulation, the cation or anion was fixed at the center of the simulation cell [30]. The cell radius $r_{0}$ can be arbitrary, as long as $r_{0}$ is large enough so that the cluster-cell interaction is negligible. For the given density of supersaturated vapor $\rho$ (see Table I), we chose $r_{0}=15 \AA$ [31]. Molecules were displaced, rotated, inserted, or removed according to the rules of the grand canonical ensemble. The probability distribution of the cluster of size $i$ was obtained by averaging over frequency of appearance of the cluster in the cell. The sampling of frequency was recorded in every $100 \mathrm{MC}$ cycles. After $1000000 \mathrm{MC}$ cycles, the simulation was stopped provided that the least frequent cluster in each interval had been sampled 100000 times.

In Figs. 1(a) and 1(b) the calculated free energy of cluster formation $\Delta G_{i}^{s} / k_{B} T$ is plotted as a function of $i$ for a supersaturated TIP4P water vapor at $298.15 \mathrm{~K}$ and OPLS methanol $\left(\rho=0.4 \times 10^{-5} \AA^{-3}\right)$ at $275.0 \mathrm{~K}$. Results for SPC/E and Dang-Chang polarizable water at $298.15 \mathrm{~K}$ are shown in Figs. 1(c) and 1(d). The barrier height to the cation- and anion-induced nucleation, $\Delta G_{+}^{*}$ and $\Delta G_{-}^{*}$, is given by the difference between the maximum and the minimum on the curve. Figures 1(a), 1(c), and 1(d) clearly show that the anion yields a lower barrier $\left(\Delta G_{-}^{*}\right)$ than cation ( $\left.\Delta G_{+}^{*}\right)$ for water, whereas Fig. 1(b) shows the opposite; namely, anion yields a higher barrier than cation for methanol. Consequently, we conclude that water has a negative sign preference, whereas methanol has a positive sign preference. Note that the quantitative difference in values of $\delta \equiv \Delta G_{-}^{*}-\Delta G_{+}^{*}$ is mainly attributed to the large difference in the equilibrium vapor densities $\rho_{0}$ (much less in liquid densities) with different water models, e.g., at $T=298.15 \mathrm{~K}$ (see Table I). Since the barrier height is very sensitive to the supersaturation $S$ or the vapor-density ratio $\rho / \rho_{0}$ [32], what we have mainly looked for is the universal qualitative trend in the quantity $\delta$, that is, the barrier-height difference due to anion and cation. Indeed, as shown in Table $\mathrm{I}, \delta$ is always negative, regardless of the model of water (SPC/E, TIP4P, Dang-Chang), the value of $\rho$, and the size and magnitude of charge of cation and anion. This leads to the general conclusion that water has a negative sign preference on condensation.

Note also that the molecular difference between water and methanol arises from the replacement of a hydrogen atom by a methyl group. How does this replacement lead to opposite sign preferences? An insightful explanation has been given by Russell [33]. Russell argued that if dipoles of water molecules on the surface of the droplet have their negative ends out of the droplet, then a negative ion in the droplet will reinforce the orientation of surface dipoles while a positive ion will reorient the surface dipoles. The latter will cause a higher free energy of droplet formation. Based on this "oriented surface dipole" argument, Russell attributed the opposite sign preference of water and methanol to the opposite orientation of the water and methanol dipole on their own surface of droplet. It
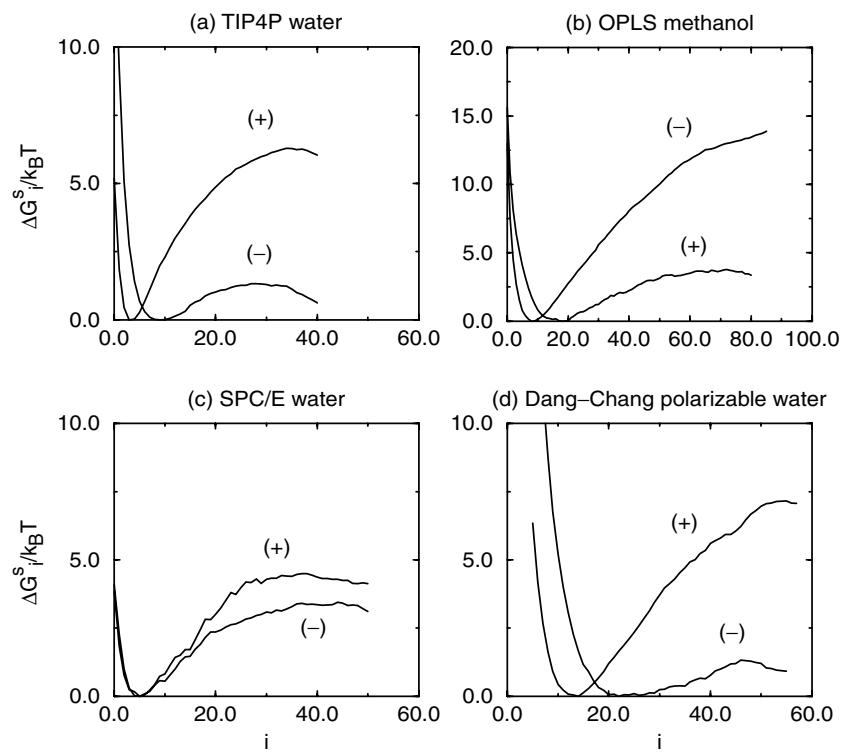

FIG. 1. Free energy of cluster formation $\Delta G_{i}^{s} / k_{B} T$ versus the size of cluster $i$ for (a) TIP4P water-0.6e ion cluster, (b) OPLS methanol-0.6e ion cluster, (c) SPC/E water-1e ion cluster, and (d) Dang-Chang polarizable water-1e ion cluster. Here $(+)$ and $(-)$ denote the cation and anion-induced nucleation, respectively. 
has been shown that Russell's argument accounts nicely for the sign preference of simple dipolar fluids [19]. However, its applicability to small water clusters requires more careful examination because several computer simulations indicate that the location of anion in water cluster appears on the surface, not at the center [4,34-36]. Moreover, there were also reports that surface dipoles of water molecules are not normal to but predominantly parallel with the surface of the cluster [37-39].

To gain more insight into the origin of the sign preference we analyzed structural characteristics of the prenucleation embryos. Figures 2(a)-2(d) display the atomic density profiles about the cation or anion for TIP4P water and methanol cluster of size 30. Figures 2(a) and 2(b) show that on the surface of ion-containing water cluster the hydrogen density is always slightly higher than the oxygen density, as in the case of pure water cluster [37,38]. However, because the hydrogen atom is much smaller than the oxygen atom, the average anion-H distance is considerably shorter than the average cation-O distance. This proximity of $\mathrm{H}$ to the anion yields more favorable ion-molecule electrostatic interactions and hence results in a negative sign preference of water. On the other hand, if an $\mathrm{H}$ atom in $\mathrm{H}_{2} \mathrm{O}$ is replaced by methyl group $\mathrm{CH}_{3}$, the resulting larger repulsive steric interactions would force the methyl group to reside on the surface of methanol cluster [40], irrespective of the sign of ion [see the density profile of methyl group in Figs. 2(c) and 2(d)]. Although the average anion- $\mathrm{H}$ distance is still much shorter than the average cation-O distance (as in the case of ion-water cluster) the orientation of the methyl group is energetically more favor-
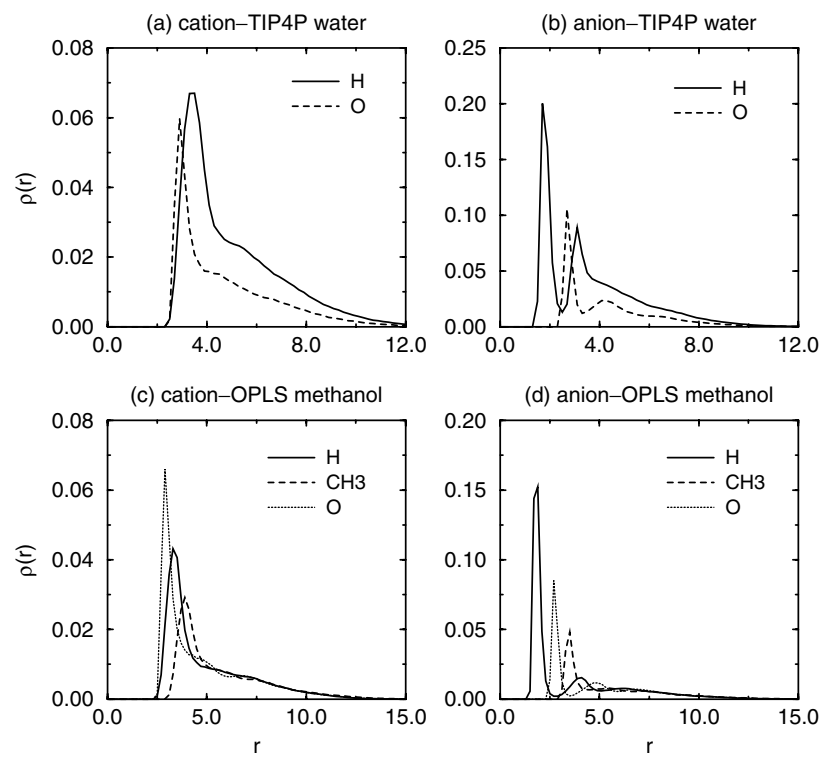

FIG. 2. Atomic density profile about the cation or anion for the prenucleation cluster of size 30: (a) cation-TIP4P water cluster, (b) anion-TIP4P water cluster, (c) cation-methanol cluster, and (d) anion-methanol cluster. The units of $r$ and $\rho(r)$ are $\AA$ and $\AA^{-3}$, respectively. able, compared to the contribution of anion-H electrostatic interaction. As a result, methanol exhibits a sign preference opposite to that of water.

The foregoing structural analysis of prenucleation embryos suggests that the opposite sign preference of water and methanol originates from a subtle competition between the intermolecular steric interaction and ionmolecular electrostatic interaction. To confirm this view we also examined energetic characteristics of the embryos. In Figs. 3(a) and 3(b) we plot the potential-energy difference (per molecule) $\Delta U=U_{\text {anion }}-U_{\text {cation }}$ versus the size of embryos $i$, for TIP4P water and methanol, respectively. The two contributions to $\Delta U$, namely, molecule-molecule interaction $\left(\Delta U_{m m}\right)$ and ion-molecule interaction $\left(\Delta U_{i m}\right)$, are also plotted as a function of

(a) TIP4P water

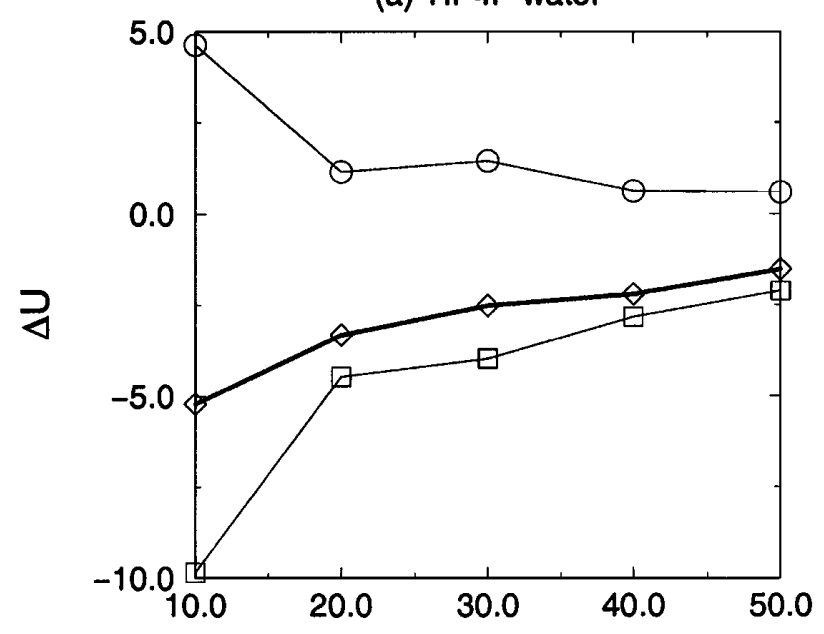

(b) OPLS methanol

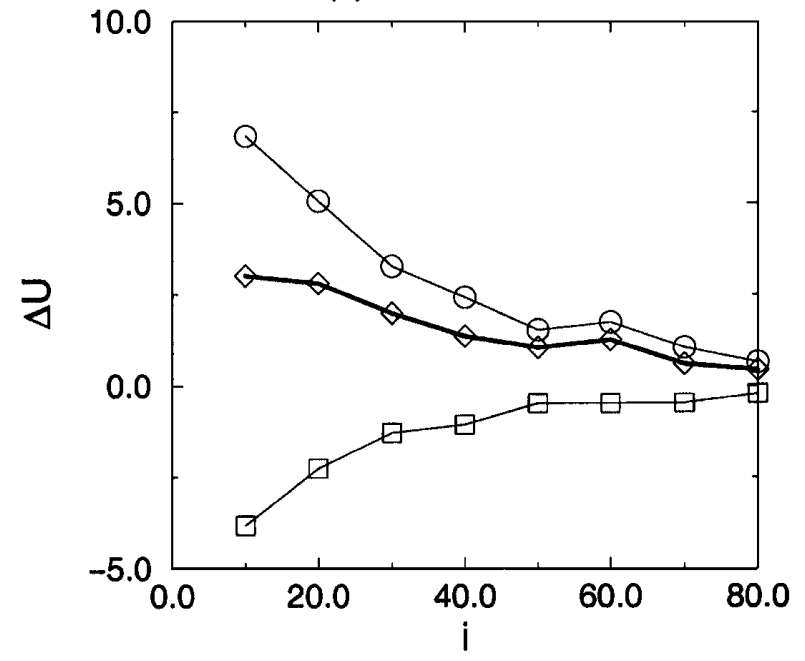

FIG. 3. The potential-energy difference $\Delta U=U_{\text {anion }}-$ $U_{\text {cation }}$ versus the size of cluster $i$ for (a) TIP4P water; (b) methanol. $\Delta U$ (thick solid line and diamonds) is a sum of molecule-molecule interaction $\Delta U_{m m}$ (solid line and circles) and ion-molecule interaction $\Delta U_{i m}$ (solid line and squares). All energies are divided by $i$, and in units of $\mathrm{kJ} / \mathrm{mol}$. 
cluster size. Indeed, $\Delta U$ is negative for water but positive for methanol, giving other evidence of the opposite sign preference for water and methanol. The absolute value of $\Delta U$, however, becomes smaller as the size of embryo increases. Figures 3(a) and 3(b) also show that for water the sign of $\Delta U$ is mainly controlled by $\Delta U_{i w}$, whereas for methanol it is controlled by $\Delta U_{m m}$. This difference in potential-energy contribution reinforces the view that the sign preferences of hydrogen-bonding molecules originate from a subtle competition between steric and electrostatic interactions. In contrast, the sign preference of simple nonpolar molecules (e.g., $\mathrm{CS}_{2}$ and $\mathrm{CH}_{4}$ ) is mainly dictated by the bare ion-molecule interaction [41], whereas the sign preference of simple anisotropic and dipolar molecules is mainly controlled by the anisotropic steric interaction [19].

We are grateful to Professor D. J. Diestler, Professor M. S. El-Shall, and Professor I. Kusaka for valuable discussions. X. C. Z. thanks the National Science Foundation, the Office of Naval Research, and the Petroleum Research Fund of the American Chemical Society for support.

[1] A. W. Castleman, Jr., I. N. Tang, and H. R. Munkelwitz, Science 173, 1025 (1971).

[2] F. Q. Yu and R. P. Turco, Geophys. Res. Lett. 27, 883 (2000).

[3] J. H. Seinfeld, Science 288, 285 (2000).

[4] E. M. Knipping, M. J. Lakin, K. L. Foster, P. Jungwirth, D. J. Tobias, R. B. Gerber, D. Dabdub, and B. J. FinlaysonPitts, Science 288, 301 (2000).

[5] C. P. Petersen and M.S. Gordon, J. Phys. Chem. A 103, 4162 (1999).

[6] C. T. R. Wilson, Philos. Trans. R. Soc. London Ser. A 189, 265 (1897).

[7] L. B. Loeb, A. F. Kip, and A. W. Einarsson, J. Chem. Phys. 6, 264 (1938).

[8] L. Scharrer, Ann. Phys. 35, 619 (1939).

[9] H. Rabeony and P. Mirabel, J. Phys. Chem. 91, 1815 (1987).

[10] M. Adachi, K. Okumura, and J. H. Seinfeld, J. Aerosol Sci. 23, 327 (1992).

[11] F. He and P. K. Hopke, J. Chem. Phys. 99, 9972 (1993).

[12] J. L. Katz, J. A. Fisk, and V. M. Chakarov, J. Chem. Phys. 101, 2309 (1994).

[13] D. Kane, G. M. Daly, and M. S. El-Shall, J. Phys. Chem. 99, 7867 (1995).

[14] D. W. Oxtoby, Acc. Chem. Res. 31, 91 (1998).

[15] J. J. Thomson, Conduction of Electricity Through Gases (Cambridge University Press, Cambridge, 1906).

[16] M. Volmer, Kinetik der phasenbildung (Steinkopff, Dresden, 1939).

[17] A. I. Rusanov and F. M. Kuni, J. Colloid Interface Sci. 100, 264 (1984).

[18] I. Kusaka, Z.-G. Wang, and J. H. Seinfeld, J. Chem. Phys. 102, 913 (1995).

[19] V. Talanquer and D. W. Oxtoby, J. Chem. Phys. 103, 3686 (1995).
[20] B. Yang, D. E. Sullivan, and C. G. Gray, J. Phys. Condens. Matter 6, 4823 (1994).

[21] B. Senger, P. Schaaf, D. S. Corti, R. Bowles, J.-C. Voegel, and H. Reiss, J. Chem. Phys. 110, 6421 (1999).

[22] K. J. Oh and X. C. Zeng, J. Chem. Phys. 112, 294 (2000).

[23] I. Kusaka, Z.-G. Wang, and J. H. Seinfeld, J. Chem. Phys. 108, 3416 (1998).

[24] W. L. Jorgensen, J. Chandresekhar, J. D. Madura, R. W. Impey, and M. L. Klein, J. Chem. Phys. 79, 926 (1983).

[25] W. L. Jorgensen, J. Phys. Chem. 90, 1276 (1986).

[26] Specifically, we chose the LJ parameters of $\mathrm{K}^{+}$reported in [J. Aqvist, J. Phys. Chem. 94, 8021 (1990)].

[27] H. J.C. Berendsen, J.R. Grigera, and T.P. Straatsma, J. Phys. Chem. 91, 6269 (1987).

[28] L. X. Dang and T.-M. Chang, J. Chem. Phys. 106, 8149 (1997).

[29] G. T. Gao, K. J. Oh, and X. C. Zeng, J. Chem. Phys. 110, 2533 (1999).

[30] The center of the simulation cell can also be fixed on the center of mass of the cluster instead of a tagged particle (ion). The calculated free energy of formation was found nearly identical for both cases.

[31] Normally, one would like to select the cell radius $r_{0}$ as large as possible in order to simulate larger critical clusters. If $r_{0}$ is too large, the efficiency of MC sampling can become an issue since very long simulation time is needed to obtain good statistics. We have examined free-energybarrier results by using the Stillinger-cluster definition (see Ref. [22]) and the Kusaka-Wang-Seinfeld-cluster definition (Ref. [23]) and found that both give almost the same results (K. J. Oh, Ph.D. thesis, 2000).

[32] This can be seen more clearly from the barrier height of homogeneous nucleation, which is expressed analytically (based on the classical theory of nucleation; see Ref. [14]), that is, $\Delta G_{c l}^{*}=16 \pi \gamma^{3} / 3\left(\rho_{l} k_{B} T \ln S\right)^{2}$, where $\gamma$ is the surface tension, $\rho_{l}$ is equilibrium liquid density at temperature $T$. Thus, if the same supersaturated-vapor density $\rho$ is used, $S$ will be different, and so will the barrier height. If the same supersaturation $S$ is used by adjusting $\rho$, the difference, though relatively small with different water models, in interfacial properties (e.g., $\gamma$ ) can also lead to different barrier height. This simple analysis indicates that it is unlikely to attain the same barrier height with different water models using either fixed vapor-density or fixed supersaturation condition.

[33] K. C. Russell, J. Chem. Phys. 50, 1809 (1969).

[34] M. A. Wilson and A. Porohille, J. Chem. Phys. 95, 6005 (1991).

[35] L. Perera and M. L. Berkowitz, J. Chem. Phys. 96, 8288 (1992).

[36] W. L. Jorgensen and D. L. Severance, J. Chem. Phys. 99, 4233 (1993).

[37] F. F. Abraham, J. Chem. Phys. 61, 1221 (1974).

[38] M. R. Mruzik, Chem. Phys. Lett. 48, 171 (1977).

[39] V.V. Zakharov, E. N. Brodskaya, and A. Laaksonen, J. Chem. Phys. 107, 10675 (1997).

[40] D. Wright and M. S. El-Shall, J. Chem. Phys. 105, 11199 (1996).

[41] I. Kusaka, Z.-G. Wang, and J. H. Seinfeld, J. Chem. Phys. 103, 8993 (1995). 